\title{
Analysis on Setup Variation According to Megavoltage Computed Tomography System
}

\author{
Sun-Yung Kim ${ }^{1}$, Hwa-Sun Kim², and Hae-Kag Lee ${ }^{3 *}$ \\ ${ }^{1}$ Center for Proton Therapy, National Cancer Center, Ilsan 10408, Korea \\ ${ }^{2}$ Department of Radiological Technology, Ansan University, Ansan 15328, Korea \\ ${ }^{3}$ Department of Computer Science and Engineering, Soonchunhyang University, Asan 31538, Korea
}

(Received 15 July 2016, Received in final form 1 September 2016, Accepted 1 September 2016)

\begin{abstract}
The aim of this study was to measure the setup variation for $X$ (lateral), $Y$ (longitude), and $Z$ (vertical) by taking magnetic megavoltage computed tomography (MVCT) before treating the brain, oropharynx, lung, and prostate patients on helical tomotherapy. In this study, 30 patients were chosen for each of the treatment areas, and their skin was labeled with a mark on a treatment planning reference point when taking CT. We preceded MVCT prior to tomotherapy and then conducted an auto registration based on the bony landmarks; image registration was used for automatically matching the patient's setup. Lastly, we confirmed and evaluated the translation coordinates of the images for 30 patients. The following shows the comparison result of the setup errors of each part: $X$ (lateral) showed the highest setup errors with $3.44 \pm 2.05$ from Lung; Y (longitude) showed the highest setup errors showing $3.40 \pm 2.87 \mathrm{~mm}$ from Prostate; and $Z$ (vertical) showed the highest setup errors showing $6.62 \pm 4.38 \mathrm{~mm}$ from Lung. This result verifies that the setup error can be prevented by taking MVCT before the treatment, and Planning Target Volume (PTV) margins can be reduced by referring to the resulting value of each treatment part. Ultimately, the dosage of the normal organs can be decreased as well as any side effects.
\end{abstract}

Keywords : MVCT, setup variation, tomotherapy

\section{Introduction}

An effective radiation therapy releases a high dose of radiation to tumor and as low radiation level as possible to the normal organs. With the advancement of computer science, not only the calculation algorithm of radiation dose has been developed, but also a more precise imagebased radiation therapy have become available by applying diagnostic image techniques such as computed tomography (CT) [1]. Furthermore, we are able to perform a radiation therapy after verifying the locations of organs around tumor and the tumor itself, by using an image that we obtain from the patient right before a radiation treatment. Besides, we can also control the amount of radiation by loading linear accelerator inside the gantry, which is the same as diagnostic CT machine [2]. The linear accelerator of tomotherapy makes $3.5 \mathrm{MV}$

(C)The Korean Magnetics Society. All rights reserved.

*Corresponding author: Tel: +82-41-530-1276

Fax: +82-41-530-1548, e-mail: lhk7083@hanmail.net of photon to obtain an MVCT image before radiation therapy and $6 \mathrm{MV}$ (mega voltage) of photon for the treatment. The most obvious difference between tomotherapy and other radiation treatments is that tomotherapy has MVCT equipment that uses 3.5 MV of photon. We can use the MVCT image to straighten a patient's pose or location before the treatment. In this way, we can minimize an inter-fractional set-up error day to day. Furthermore, when planning a treatment with an MVCT image, we are able to perform a stereotactic radiation treatment by confirming a planned dosage and assessing the changes of normal organs and tumor [3]. However, the image quality of MVCT is not as good for distinguishing the soft tissues from low-contrast resolution [3, 4]. Therefore, when it comes to parenchymal tumor, it is difficult to distinguish the soft tissues from mediastinal tumor even when we can find the location and size of tumor with the MVCT images [5]. Besides, since we can see the changes of normal tissues and tumor and reproduce the dose distribution with tomotherapy, we should change the treatment plan if there are huge changes in the 
structure, such as the lighter weight of patient or the smaller size of tumor $[1,6]$. Sometimes in a real clinic, some anatomical changes may occur due to the decrease in tumor size or the weight loss in the patients with head and neck cancer [4]. Therefore, this study aimed to measure the setup variation of $X$ (lateral), Y (longitude), and $\mathrm{Z}$ (vertical) by taking mega voltage computed tomography (MVCT) before treating the brain, oropharynx, lung, and prostate in the patients on Helical Tomotherapy.

\section{Subjects and Method}

In this study, we computed the setup errors of the brain, oropharynx, lung, and prostate of each 30 patients who were being treated by tomotherapy image registration (Table 1). Before we started the treatment, all patients took kilovoltage computed tomography (KVCT) for treatment planning. The patients were placed in a headfirst supine position. For the brain and oropharynx patients, we used Aquaplaster (Med-Tec, Orange City, IA) immobilization device. In the case of lung patients, the patients held hands to wing-board (Med-Tec, Orange City, IA); and for prostate patients, the prostate was fixed by placing a $100 \mathrm{cc}$ of rectal balloon into the rectum and filling bladder. When taking CT, we marked the reference points for the treatment plan by locating 3 lead points on the right, left, and upper side, based on the patient's body surface. These points were used as the coordinates for tomotherapy treatment setup's daily CT reference points $\mathrm{X}$ (lateral), Y (longitude), Z (vertical) $(0,0,0)$. We took a Spiral KVCT image with a $3 \mathrm{~mm}$ axial slice, Pitch 1.7, and the image was used to contour the critical organ structure and for planning the target volume (PTV) by Treatment Planning System (Philips Medical System,
Eindhoven, The Netherlands). We also sent the image to tomotherapy planning by using digital imaging and communications in medicine-radiation therapy (DICOM-RT) protocol. Prior to each treatment, the patients were placed in the same position as when they used the fixation machine from CT simulation, and the lasers of infirmary was matched to the coordinate of reference points $(0,0$, 0 ). We took MVCT with a $6 \mathrm{~mm}$ of slice thickness, preceded automatic bone registration, and then performed manual image registration based on PTV (Fig. 1). We reported X (lateral), Y (longitude), and Z (vertical) of MVCT scan image, treatment planning KVCT image, as well as setup errors, which were compared and analyzed by using each coordinate's movement value. Also, a precise treatment set-up was done by verifying the difference in dose distribution (Fig. 2). In order to conduct a statistical analysis, simple sampling T-test was used to compare X (lateral), Y (longitude), and Z (vertical) of MVCT scan image and treatment planning KVCT image with setup errors. Furthermore, the average value of setup errors was compared by using ANOVA (SPSS Ver. 18.0, USA, Chicago) test from each part.

\section{Result}

For the brain, setup errors of $X$ (lateral) was $1.69 \mathrm{~mm}$, $\mathrm{Y}$ (longitude) was $3.14 \mathrm{~mm}$, and $\mathrm{Z}$ (vertical) was 4.15 $\mathrm{mm}$; and $\mathrm{Z}$ (vertical) showed the highest setup error. For the head and the neck, setup errors $X$ (lateral) was 3.35 $\mathrm{mm}$, Y (longitude) was $1.50 \mathrm{~mm}$, and $\mathrm{Z}$ (vertical) was $4.45 \mathrm{~mm}$; and $\mathrm{Z}$ (vertical) showed the highest setup error. For the lung, the setup errors of $X$ (lateral) was $3.44 \mathrm{~mm}$, $\mathrm{Y}$ (longitude) was $2.55 \mathrm{~mm}$, and Z (vertical) was 6.62 $\mathrm{mm}$; and Z (vertical) showed the highest setup error. For

Table 1. Patient characteristics.

\begin{tabular}{lc}
\hline \hline No. of patients & Brain (30), Oropharynx (30), Lung (30), Prostate (30) \\
Male/female & Brain (17/13), Oropharynx (23/7), Lung (30), Prostate (30) \\
Age (y), mean (range) & 61.3 (29-79) \\
Pathology, n (\%) & \\
Adenocarcinoma & Brain (3), Lung (19), Prostate (30) \\
Squamous cell carcinoma & Brain (5), Oropharynx (30), Lung (11), \\
Glioblastoma & Brain (8) \\
Melanoma & Brain (10), \\
Astrocytoma & Brain (4), \\
Stage, n (\%) & \\
I 0 & \\
II 1 (10) & Brain (8), Oropharynx (8), Lung (3), Prostate (30) \\
III 7 (70) & Brain (20), Oropharynx (18), Lung (27), \\
IV 2 (20) & Brain (2), Oropharyn (4), \\
Total radiation dose (fractions) & Brain 50Gy (20), Oropharynx 60Gy (30), Lung 60Gy (25), Prostate 65Gy (25) \\
\hline
\end{tabular}



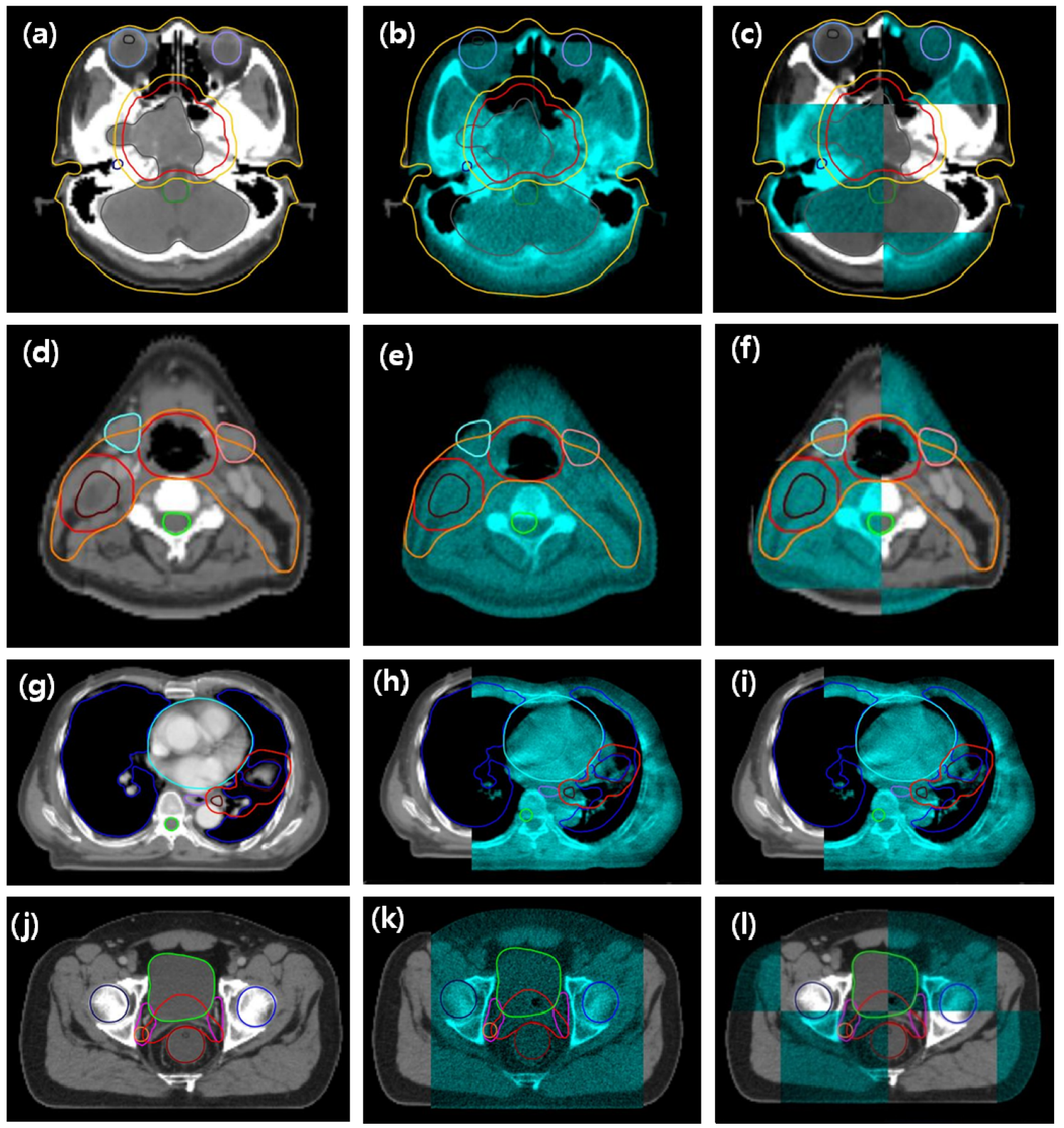

Fig. 1. (Color online) KVCT CT Image in brain (a), MVCT Tomo image (b), and Automatic registration image (c). KVCT CT Image in head\&neck (d), MVCT Tomo image (e), and Automatic registration image (f). KVCT CT Image in lung (g) MVCT Tomo image (h), and Automatic registration image (i). KVCT CT Image (j), MVCT Tomo image in prostate (k), and Automatic registration image (1).

the prostate, the setup errors of $X$ (lateral) was $3.10 \mathrm{~mm}$, $\mathrm{Y}$ (longitude) was $3.40 \mathrm{~mm}$, and $\mathrm{Z}$ (vertical) was 3.46 $\mathrm{mm}$; and $\mathrm{Z}$ (vertical) showed the highest setup error. For comparing the setup errors of each part, $\mathrm{X}$ (lateral) showed the lowest setup errors in the brain, with $1.69 \pm 1.65$ $\mathrm{mm}$; and it showed the highest setup errors in the lung, with $3.44 \pm 2.05 \mathrm{~mm}$. On the other hand, Y (longitude) showed the lowest setup errors in the head and neck, with $2.29 \pm 2.12 \mathrm{~mm}$; and it showed the highest setup errors in prostate, with $3.40 \pm 2.87 \mathrm{~mm}$. Also, Z (vertical) showed the lowest setup errors in the brain, with $4.15 \pm 2.29 \mathrm{~mm}$; and it showed the highest setup errors in the lung, with $6.62 \pm 4.38 \mathrm{~mm}(\mathrm{p}<0.05)$ (Table 2). In conclusion, $X$ (lateral) showed the highest setup errors in lung; Y (longitude) showed the highest setup errors in prostate; and $\mathrm{Z}$ (vertical) showed the highest setup errors in lung.

\section{Discussion}

This study aimed to find if there is a difference value in 

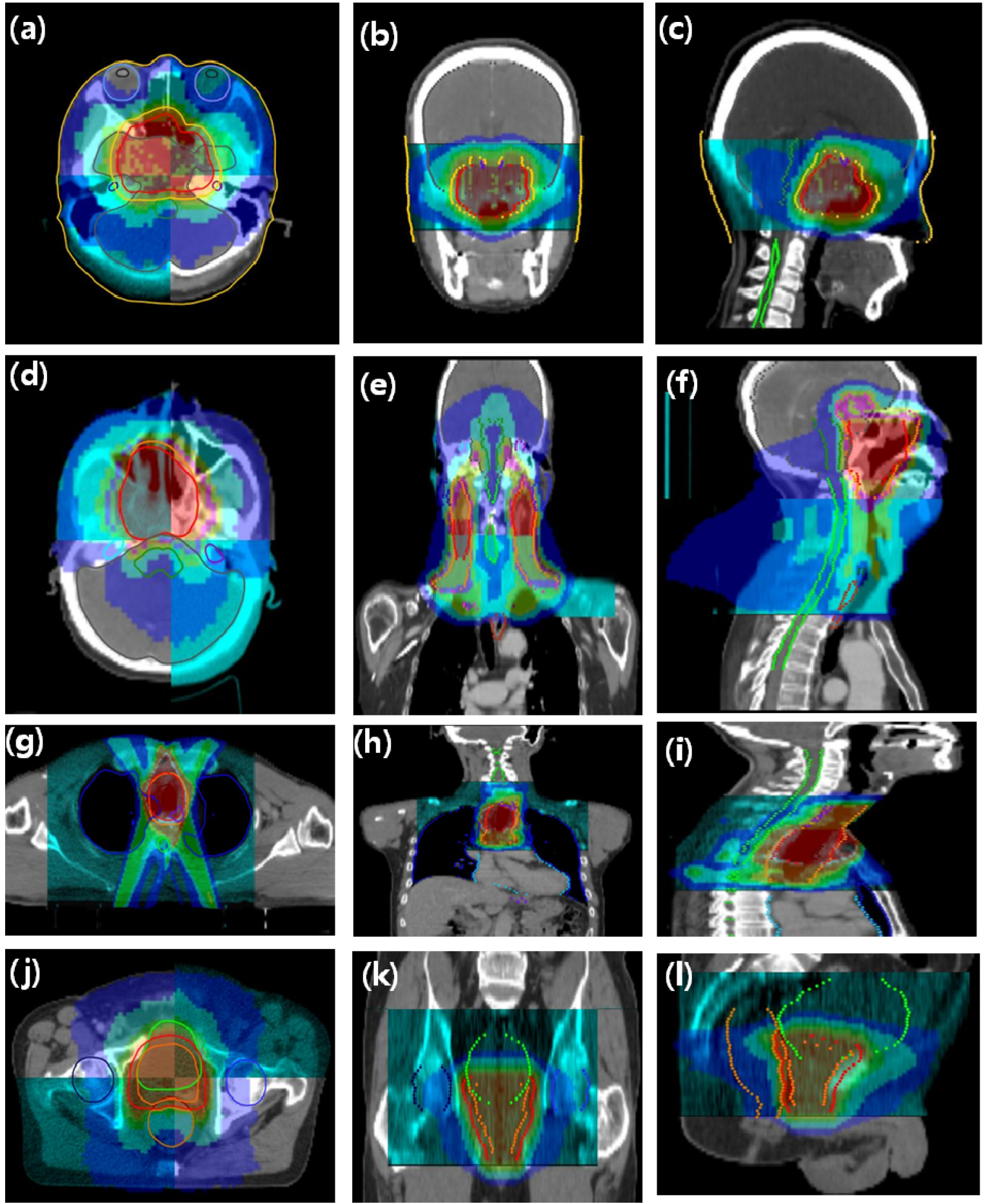

Fig. 2. (Color online) Axial (a), Coronal (b), and sagittal dose distribution in tomo image from the brain (c). Axial (d), Coronal (e), Sagittal dose distribution tomo image from the head\&neck (f). Axial (g), Coronal (h), and Sagittal dose distribution tomo image from the lung (i). Axial (j), Coronal (k), and Sagittal dose distribution tomo image from the prostate (l).

the treatment area being adapted to the tomotherapy treatment, when taking a CT after setting up treatment plan in the same way as the actual treatment. Also, the most ideal planning target volume (PTV) margins was found for planning a treatment. Since we used Aquaplaster (Med-Tec, Orange City, IA) immobilization Device for the brain and the head\&neck (oropharynx), which has great reproducibility for fixing patients, we had a low 
Table 2. MVCT scan images of Brain, Oropharynx, Lung, Prostate; X (lateral), Y (longitude), Z (vertical) of the treatment planning KVCT images; and numerical value of setup errors.

\begin{tabular}{|c|c|c|c|c|c|c|c|c|c|c|}
\hline \multirow{2}{*}{ Region } & \multicolumn{3}{|c|}{ Reference point } & \multicolumn{3}{|c|}{1 day } & \multicolumn{3}{|c|}{ Average difference } & \multirow{2}{*}{$\mathrm{p}$} \\
\hline & X (lateral) & Y (longitud) & $\mathrm{Z}$ (vertical) & X (lateral) & Y (longitud) & Z (vertical) & X (lateral) & Y (longitud) & $\mathrm{Z}$ (vertical) & \\
\hline Brain & 0 & 0 & 0 & $1.69 \pm 1.65$ & $3.14 \pm 2.02$ & $4.15 \pm 2.29$ & 1.69 & 3.14 & 4.15 & 0.00 \\
\hline Head\&Neck & 0 & 0 & 0 & $3.35 \pm 2.27$ & $2.29 \pm 2.12$ & $5.23 \pm 2.08$ & 3.35 & 1.50 & 4.45 & 0.00 \\
\hline Lung & 0 & 0 & 0 & $3.44 \pm 2.05$ & $2.55 \pm 2.35$ & $6.62 \pm 4.38$ & 3.44 & 2.55 & 6.62 & 0.00 \\
\hline Prostate & 0 & 0 & 0 & $3.10 \pm 2.34$ & $3.40 \pm 2.87$ & $4.52 \pm 2.84$ & 3.10 & 3.40 & 3.46 & 0.00 \\
\hline
\end{tabular}

variability in the set-up. However, for the head\&neck (Oropharynx), there was weight decrease in the patients due to the damage of treatment area during the treatment, which moved the patients away from the fixation tool. This resulted in a low reproducibility compared to the brain and the high set-up variability. For the lung area, the set-up variability can cause a huge resulting value depending on the breaths the patients take. For the prostate, unlike other treatments it has the highest set-up variability due to the declined reproducibility, since it uses bladder filling volume and rectum balloon. As a result, lung area was found to have the highest set-up variability. Generally, for treating thoracoabdominal patients with radiation, the movement from the breathing makes it hard to deliver a precise amount of dose to the gross tumor volume (GTV), and it affect the overall treatment due to the damages on the normal tissues [7]. According to the International Commission on Radiological Protection (ICRU)'s report 62, the intrafraction uncertainties resulted in the uncertainties of treatment; and when these occurred, it is better to consider the setup margin when fixing the subject [8]. With the advancement of image-guided radiation therapy (IGRT) that reduces the uncertainties of treatment, respiratory gating therapy uses respiration cycle and tumor tracking radiation therapy accounts for the movement of tumor [9]. Respiratory gating therapy is a radiation therapy performed in a set-up cycle by using respiration-monitoring equipment $[10,11]$. However, tomotherapy (Hi-Art II, TomoTherapy, USA) radiation treatment is unable to reflect the movement of tumor and respiration cycle. Tomotherapy is a treatment that combined helical computed tomography and linear accelerator (LINAC), and it uses mega voltage computed tomography (MVCT) to detect and correct the location of tumor before the treatment [12-17]. But, in order to minimize the movement of organs due to respiration, we can only depend on the patient fixation tool. Meanwhile, in performing a radiation therapy for prostate cancer, the rectal balloon filled with air is being used for the target reproducibility and to protect the rectum. This rectal balloon is used for minimizing the rectum expansion for physio- logical reasons, and it creates a distance between the rear wall of rectum and prostate $[18,19]$. It is well known that a dose perturbation occurs if air was included during the radiation therapy. Even though we have been using the fixing equipment to reduce the setup errors when performing tomotherapy treatment, there were setup errors just like this study.

\section{Conclusion}

We should revise the numeric value of setup errors when planning a radiation therapy for the brain, head\& neck, lung, and prostate tumor patients. If we take MVCT before every treatment, we can prevent setup errors; and if we refer to the result value of each treatment area and plan a treatment, we can reduce planning target volume (PTV). As a result, we will be able to reduce the dosage of normal organs and minimize the side effects.

\section{Acknowledgement}

Sun-Yung Kim and Hwa-Sun Kim equally contributed to this work. They are co-authors of first.

This work was supported in part by the Soonchunhyang University Research Fund.

\section{References}

[1] T. R. Mackie, Phys. Med. Biol. 51, R427 (2006).

[2] G. L. Whitelaw, I. Blasiak-Wal, K. Cooke, C. Usher, N. D. Macdougall, and P. N. Plowman, Br. J. Radiol. 81, 333 (2008).

[3] W. A. Tome, H. A. Jaradat, I. A. Nelson, M. A. Ritter, and M. P. Mehta, Front Radiat Ther Oncol. 40, 162 (2007).

[4] K. C. Keum, J. Korean Med. Assoc. 51, 619 (2008).

[5] J. S. Welsh, K. Bradley, K. J. Ruchala, T. R. Mackie, R. Manon, R. Patel, P. Wiederholt, M. Lock, S. Hui, and M. P. Mehta, Clin Lung Cancer. 205, 303 (2004).

[6] J. S. Welsh, M. Lock, P. M. Harari, W. A. Tome', J. Fowler, T. R. Mackie, M. Ritter, J. Kapatoes, L. Forrest, R. Chappell, B. Paliwal, and M. P. Mehta, Technol Can- 
cer Res Treat. 5, 465 (2006)

[7] C. W. Stevens, R. F. Munden, K. M. Forster, J. F. Kelly, Z. Liao, G. Starkschall, S. Tucker, and R. Komaki, Int. J. Radiat. Oncol. Biol. Phys. 51, 62 (2001).

[8] ICRU Report 62, Prescribing, recording and reporting photon beam therapy. International Commission on Radiation Units and Measurements, Supplement to ICRU Report 50, 1999.

[9] J. H. Jung, K. H. Cho, J. W. Lee, M. J. Kim, K. C. Lim, S. K. Moon, Y. H. Kim, and T. S. Suh, J. Med. Phys. 22, 72 (2011).

[10] Y. G. van der Geld, B. van Triest, W. F. Verbakel, J. R. van Sörnsen de Koste, S. Senan, B. J. Slotman, and F. J. Lagerwaard, Int. J. Radiat. Oncol. Biol. Phys. 72, 1215 (2008).

[11] A. A. Garsa, R. S. Andrade, D. E. Heron, S. Beriwal, H. Kim, E. Brandner, G. Kuo, H. Chen, K. Gerszten, J. N. Yue, M. S. Huq, J. Lee, R. Lalonde, and A. Wu, Int. J. Gynecol. Cancer. 17, 55 (2007).

[12] T. R. Mackie, T. Holmes, S. Swerdloff, P. Reckwerdt, J.
O. Deasy, J. Yang, B. Paliwal, and T. Kinsella, Med. Phys. 20, 1709 (1993).

[13] T. R. Mackie, J. Kapatoes, K. Ruchala, W. Lu, C. Wu, G. Olivera, L. Forrest, W. Tome, J. Welsh, R. Jeraj, P. Harari, P. Reckwerdt, B. Paliwal, M. Ritter, H. Keller, J. Fowler, and M. Mehta, Int. J. Radiat. Oncol. Biol. Phys. 56, 89 (2003).

[14] M. T. Kim, H. K. Lee, Y. C. Heo, and J. H. Cho, J. Magn. 19, 15 (2014).

[15] Y. C. Heo, H. K. Lee, C. S. Park, and J. H. Cho, J. Magn. 20, 40 (2015).

[16] J. H. Lee, H. K. Lee, J. H. Cho, and M. J. Cheon, J. Magn. 19, 372 (2014).

[17] J. H. Cho, J. H. Lee, C. S. Park, S. Y. Lee, J. Lee, D. H. Moon, and H. K. Lee, J. Magn. 19, 248 (2014).

[18] J. S. Song, L. E. Court, and R. A. Cormack, Med. Dosim. 32, 151 (2007).

[19] R. J. Smeenk, B. S. Teh, E. B. Butler, E. N. van Lin, and J. H. Kaanders, Radiother Oncol. 9, 277 (2010). 